\title{
Advance in Etiology and Rehabilitation Treatment of Patellofemoral Pain (Review)
}

\author{
Dong Qiu ${ }^{1}$, Zha Zhengang ${ }^{1}$
}

${ }^{1}$ Center for joint and sports medicine, The First Affiliated Hospital of Jinan University, Guangzhou, China

\begin{abstract}
Patellofemoral pain (PFP) is the most common chronic musculoskeletal disease seen in sports medicine clinics. The pathogenesis of PFP is not yet clear, but there have been many biomechanical and pathophysiological studies related to PFP. These studies have further improved the understanding of PFP and aided in its treatment. Due to the high prevalence, long course and difficulty in eradicating PFP, most treatment options to date still aim to improve symptoms and have not resulted in an effective treatment approach. Reasonable rehabilitation therapy can relieve pain, improve motor function and help patients regain confidence in life and work. Therefore, further research into the pathogenesis of PFP and the search for a set of standardized and effective rehabilitation methods for PFP are particularly important in the future.
\end{abstract}

Keywords: Patellofemoral Pain, Pathogenesis, Rehabilitation Treatment

\section{Introduction}

Patellofemoral pain (PFP) is a diffuse pain that occurs in the peripatellar or retropatellar region and can be triggered or exacerbated by weight-bearing knee flexion activities (squatting, climbing stairs, running or jumping) (Crossley et al. 2016). In recent years, with the construction of a "Healthy China" and the "National Fitness" boom, running has become increasingly popular, and marathons have become an important way for the general public to actively participate in exercise. While running can improve cardiorespiratory fitness, it can also increase the risk of musculoskeletal injury, with PFP being the most common form of injury. The latest research shows that the prevalence of PFP is as high as $20 \%$ in young adults in China (Xu et al. 2018). PFP is one of the more difficult musculoskeletal disorders to treat today, with a lack of effective treatment and medication that provides only short-term relief of pain and eventually progresses to patellofemoral arthritis, which inevitably requires surgery. PFP not only places a significant financial burden on society, but also has a significant impact on patients' quality of life and psychological well-being, as well as the potential for disability, if not treated promptly and effectively. Therefore, it is important to provide long-term rehabilitation exercises to reduce complications. The present review of the etiology of PFP and the latest advances in rehabilitation treatment is intended to provide new ideas and approaches for future research and treatment options.

\section{New Insights into the Etiology of PFP Abnormal Patellofemoral Trajectory}

The more positive the trajectory of the patella in the femoral trochlea, the greater the contact area of the patellofemoral joint, the more evenly the stresses are distributed, and the lower the stresses on the patellofemoral joint surface. When the patellar trajectory is abnormal, the contact area between the patellar surface and the femoral trochlea decreases and the stress on the patellofemoral joint surface increases accordingly, resulting in PFP when the stress exceeds the maximum range that the patellofemoral joint can withstand (Liao et al. 2018). Wilson (Wilson et al. 2009) system examined the patellar trajectory of young PFP patients during standing and squatting and found that the patella was significantly more laterally deflected, rotated and had a tendency to increase lateral tilt in PFP patients compared to healthy controls, with poor patellar trajectory most evident when squatting while bearing weight. Similarly, a systematic review that included 40 studies noted that increased lateral patellar displacement on MRI, increased patellar tilt angle, and other indicators of abnormal patellar trajectory were associated with PFP (Macri et al. 2016). In addition, other studies have found that greater patellar tilt is associated with greater pain and that abnormal patellofemoral trajectory leads to progression of patellofemoral arthritis (Hunter et al. 2007). Specifically, lateral displacement is strongly associated with the development of lateral patellofemoral arthritis, whereas medial displacement accelerates the progression of medial patellofemoral arthritis. Of course, most of the current studies are observational and the causal relationship between abnormal patellofemoral trajectory and PFP still needs further investigation.
Muscle Function Abnormalities Muscle Strength Abnormalities
A recent Meta-analysis identified quadriceps weakness as an independent risk factor for PFP (Neal et al. 2019). It was found that patients with PFP commonly have diminished or inhibited activation of the medial femoral muscle and that medial femoral muscle weakness has the greatest impact of any of the quadriceps (Gaitonde et al. 2019). Theoretically, this imbalance in quadriceps muscle strength could further lead to abnormal patellar trajectory, increased patellofemoral joint stress and the development of 
PFP (Karst et al. 1995). Pal et al. (Pal et al. 2019) used data provided by high-resolution MRI to develop a finite element model of stair walking up and down stairs in patients with PFP and found that the interpatellofemoral joint stress is most sensitive to changes in medial femoral muscle strength, but the exact mechanism by which medial femoral muscle weakness leads to PFP remains unknown.

In addition, muscle strength abnormalities in the hip joint have been implicated in the development of PFP. For example, a prospective cohort study by Boil et al (Boling et al. 2009) of 1597 recruits showed that greater external rotation strength of the hip joint was associated with the development of PFP. However, a prospective study by Finnoff et al. (Finnoff et al. 2011) of 98 high school runners showed that greater hip abduction strength and lesser external rotation strength were associated with the development of patellofemoral joint pain. In contrast, Thijs et al (Thijs et al. 2011) concluded that hip muscle strength was not directly related to the risk of developing PFP. Notably, Ramskov et al. (Ramskov et al. 2015) conducted a year-long prospective study of 629 novice runners and found no clear correlation between hip strength and the development of PFP at running distances below $50 \mathrm{~km}$, but stronger hip abduction strength at running distances above $50 \mathrm{~km}$ reduced the probability of PFP However, stronger hip abduction strength at running distances above $50 \mathrm{~km}$ reduced the probability of PFP. In view of this, further high-quality studies are needed to explore the specific relationship between hip muscular strength and PFP and the mechanisms involved.

\section{Reduced Muscle Flexibility}

Reduced lower limb flexibility can also lead to abnormal patellar trajectories, and White et al (White et al. 2009) compared lower limb muscle tone in patients with PFP and normal controls with passive knee extension and found that lower limb muscle tone was significantly higher in patients with PFP than in normal controls. However, this cross-sectional study did not confirm a causal relationship between muscle flexibility and PFP. Subsequently, Whyte et al (Whyte et al. 2010) investigated the relationship between patellofemoral joint stress and lower limb muscle flexibility during weight-bearing activities and found that muscle tension around the knee joint led to a reduction in patellofemoral joint contact area and an increase in patellofemoral joint stress, which led to the This change was particularly evident in knee flexion at $60^{\circ}$. In an in vitro study, hamstring tension was found to increase patellar flexion, valgus, and valgus by approximately $1^{\circ}, 0.5^{\circ}$, and $0.2 \mathrm{~mm}$, respectively, and to increase total lateral patellar stress by approximately $5 \%$ during knee flexion and extension activities, resulting in abnormal patellar trajectories (Elias et al. 2011). In recent years, an increasing number of clinicians have added training programmes to improve lower limb flexibility to their rehabilitation programmes for patients with PFP, with good results (Zago et al. 2020; Lee et al. 2021).

\section{Structural Abnormalities of the Foot}

Dynamic valgus due to structural abnormalities of the foot is closely associated with the development of PFP. Dynamic valgus is a standing position in which the knee collapses inwards due to excessive valgus, internal and external rotation or both, which increases the force of lateral deflection of the patella and ultimately leads to patellar maltracking (Schmitz et al. 2009). The available literature suggests that structural abnormalities of the foot increase internal rotation of the tibia in patients with PFP, leading to dynamic valgus. First, Barton et al (Barton et al. 2010) looked at the forefoot characteristics of patients with PFP, who had greater forefoot abduction compared to healthy controls. Subsequently, Mølgaard et al (Mølgaard et al. 2011) observed all high school students within a Danish high school and found greater navicular prolapse, drift and foot dorsiflexion in high school students with PFP, suggesting that structural abnormalities of the midfoot are an important factor contributing to PFP. More recently, Luz et al (Luz et al. 2018) found that excessive hindfoot valgus deformity may be associated with the persistence of PFP symptoms after observing 54 running athletes. Thus athletes with flat feet are prone to excessive foot rotation anteriorly and the tibia compensates by rotating inwards, thus affecting the patellofemoral joint trajectory and predisposing them to PFP.

\section{Psychological Factors}

Recently, the association between pain and knee dysfunction and psychological factors in patients with PFP has been gaining attention. For example, Priore et al (Priore et al. 2019) found that most patients with PFP experience psychological disorders such as anxiety, depression, kinesiophobia, and pain catastrophizing, and that pain catastrophizing explained $37 \%$ of patients' severe pain and depression explained $56 \%$ of functional impairment. Similarly, a recent systematic evaluation showed a linear relationship between symptom severity of PFP and psychological factors related to anxiety, depression, pain catastrophizing, and kinesiophobia (Maclachlan et al. 2017). In addition, Liam et al (Maclachlan et al. 2018) found significantly higher depression and pain catastrophizing scores in the PFP severe group than in the mild group and healthy controls. Certainly, the dialectical relationship between PFP and psychological factors still deserves further research.

\section{Advances in Rehabilitation Treatment for PFP Exercise Therapy}

The 4th edition of the International Association for the Study of PFP Expert Consensus states that exercise therapy can not only reduce pain in patients with PFP in the short term, but can also improve joint function in the medium to long term (Collins et al. 
2018). The main targets of exercise therapy include the core muscles, hip muscles and knee muscles, but specific exercise prescriptions remain controversial. Firstly, the optimal frequency of exercise remains unclear. Most current prescriptions instruct patients with PFP to perform functional exercise for 4-6 weeks, 2-4 times per week. chevidikunnan et al (Chevidikunnan et al. 2016) performed core muscle strengthening exercises 3 times per week for 4 weeks in patients with PFP and found significant improvements in symptoms and function in patients with PFP. Meanwhile, Hamstra et al (Hamstra-Wright et al. 2017) instructed 157 patients with PFP in hip and knee strengthening training for 6 weeks, 6 times per week, and found significant improvements in symptoms, function, and core strength before and after rehabilitation, and a relapse rate of only $5 \%$ over 2 years. Further research is still needed to determine whether the rehabilitation effect will improve further with increased exercise frequency. Secondly, the choice of exercise modality remains controversial. Although international guidelines clearly suggest that combined hip and knee exercises are superior to knee exercises alone (Collins et al. 2018). However, Hott et al (Hott et al. 2019) instructed 112 young patients with PFP to perform hip exercise, knee exercise and free exercise respectively, with a 3-month follow-up, and found that symptoms and function improved in all 3 groups compared to pre-exercise, and that there were no significant differences between the 3 groups. In addition, a recent Meta-analysis showed that when performing exercise rehabilitation, starting exercises with the proximal muscles resulted in greater pain reduction and improved function (Lack et al. 2015). In light of this, exercise therapy should target core muscles such as the rectus abdominis, muscles of hip abduction and external rotation, quadriceps and hamstrings, but specific exercise prescription choices need to be further validated and individualised differences fully considered.

\section{Gait Retraining}

Gait retraining is primarily indicated for patients who have developed PFP as a result of prolonged running. The International Association for the Study of PFP expert consensus states that abnormal gait in the coronal, sagittal and horizontal planes in runners often leads to poor patellar trajectory, increased patellofemoral joint stress and ultimately patellofemoral joint pain (Powers et al. 2017). Therefore, correcting the gait of runners is extremely important to prevent and treat PFP. For example, Roper et al. (Roper et al. 2016) found that instructing runners with PFP to change their running posture from rearfoot to forefoot significantly improved hip and knee mobility and improved knee pain symptoms. Similarly, Bramah et al. (Bramah et al. 2019) instructed patients with PFP to increase their stride frequency by $10 \%$ while running and observed significant increases in weekly running volume, maximum pain-free running distance, and significant improvements in pain and function in PFP runners at 4-week and 3-month follow-ups. In addition, Santos et al (Dos Santos et al. 2019) investigated the effects of three gait retraining modalities (forefoot landing, a $10 \%$ increase in stride frequency, and trunk tilt) on lower limb biomechanics and clinical symptoms in PFP runners and found that all three modalities had some clinical efficacy and all improved pain and function in PFP runners. However, incorrect running posture can cause considerable damage to the musculoskeletal system and consciously training one's gait can be effective in reducing patellofemoral joint stress and preventing PFP.

\section{Patellar Patches}

The Chinese Guidelines for the Treatment of Patellofemoral Arthritis (2020 Edition) state that patellar patches are effective in correcting patellar malposition, reducing patellofemoral joint stress and relieving painful symptoms. mechanical rearrangement in order to reduce pain and improve function in patients with PFP (McConnell et al. 2000). Kenzo then designed the Kinesio patellar patch, based on the classic patellar patch, to apply 10-15\% tension towards the patella at the start of the quadriceps and hamstrings, with the aim of correcting poor patellar trajectory and relieving muscle tension (Akbaş et al. 2011). A systematic evaluation including 11 studies found that both patellar patches positively affected pain and quality of life in patients with PFP, however, the Kinesio patellar patch increased muscle flexibility while relieving pain and improving poor patellar trajectory (Chang et al. 2015).

Although, the patellar patch is unanimously recommended by many national and international expert consensus and practice guidelines (Powers et al. 2017; Barton et al. 2015). However, the exact mechanism of action is unclear, while the follow-up period for patella patches is mostly short, and their safety and efficacy for long-term use have not been established. However, the patellar patch can be used in conjunction with other rehabilitation project and does not hinder training or competition during its use, so its use in the rehabilitation project of athlete patients can be attempted.

\section{Foot Orthoses and Special Insoles}

Structural abnormalities of the foot can lead to dynamic valgus and ultimately to PFP (Barton et al. 2010; Mølgaard et al. 2011; Luz et al. 2018). Matthews et al (Matthews et al. 2020) randomized 192 patients with PFP into a foot orthosis group and a hip muscle exercise group and found no significant difference in rehabilitation outcomes between the two at 12 weeks of follow-up, confirming that wearing foot orthoses and exercise therapy The results were equally effective. A systematic evaluation that included 11 clinical studies showed that foot orthoses 
can reduce pain and improve function in the short as well as the long term, but foot orthoses have only a slight effect on lower limb kinematics and muscle activation, and the relationship between the biomechanical effects of orthoses and pain remains unclear (Ahlhelm et al. 2015). In addition, a comparison of the effects of flat shoes and foot orthoses found that although wearing a foot orthosis for 6 weeks improved pain in patients with PFP, there was no significant difference in rehabilitation outcomes between the two at one year follow-up and there were more adverse effects such as chafing and blistering in the foot orthosis group (Hossain et al. 2011). It is worth noting that foot orthoses combined with foot-targeted exercise methods can achieve better results than knee exercise therapy alone (Mølgaard et al. 2018). This suggests that some patients with foot deformities such as valgus and pronator augmentation are more likely to benefit from foot orthoses and special insoles, and that the choice to wear foot orthoses or special insoles should be combined with other rehabilitation therapies.

\section{Electrical stimulation}

Theoretically, electrical stimulation can be used as an alternative to exercise therapy to increase muscle contraction and reduce pain. For this reason, electrical stimulation is often used as a complementary therapy to the rehabilitation of patients with PFP. Currently, the two main types of electrical stimulation include neuromuscular electrical stimulation and transcutaneous electrical nerve stimulation, both of which have been shown to be reliable and effective. Studies have shown that 15 min of electrical neuromuscular stimulation improves activation of the gluteus medius during activity and relieves pain symptoms in patients with PFP (Glaviano et al. 2016). Similarly, Son et al (Son et al. 2017) found that transcutaneous electrical stimulation was not only effective in reducing knee pain in subjects, but also in improving pain-induced gait abnormalities. In addition, Talbot et al. (Talbot et al. 2020) found that exercise therapy combined with electrical stimulation significantly increased knee mobility, but there was no significant difference in the effectiveness of the different electrical stimulation treatments. In contrast, most of the current clinical studies on electrical stimulation therapy for PFP are small trials with short follow-up periods and cannot confirm the exact role of electrical stimulation therapy in the rehabilitation of PFP (Martimbianco et al. 2017). Further research is still needed to analyze its therapeutic mechanisms and to standardize optimal treatment protocols.

\section{Conclusion}

PFP is one of the most common chronic conditions seen in sports medicine clinics, and is increasingly valued by clinicians and rehabilitation physicians both nationally and internationally, as it is mainly characterized by anterior knee pain during weight-bearing knee flexion activities. Currently, guidelines and expert consensus on PFP are being updated and many rehabilitation therapies are being used with good results in clinical practice. Unfortunately, there have been no breakthroughs in the management of PFP, and thousands of patients are or will be suffering from pain. To change this situation, future research should focus on identifying risk factors, testing effective prevention and treatment measures, developing educational strategies, assessing the impact of psychosocial factors, evaluating the effectiveness of exercise in training, and improving clinicians' assessment skills.

\section{Reference}

1. Ahlhelm A, Alfuth M. The Influence of Foot Orthoses on Patellofemoral Pain Syndrome: A Systematic Analysis of the Literature. Sportverletzung Sportschaden : Organ der Gesellschaft fur Orthopadisch-Traumatologische Sportmedizin (2015) 29(2):107-117. PMID:25675398. https://doi.org/10.1055/s-0034-1399002

2. Akbaş E, Atay A, Yüksel I. The effects of additional kinesio taping over exercise in the treatment of patellofemoral pain syndrome. Acta Orthop Traumatol Turc (2011) 45(5):335-41. PMID:22032998. https://doi.org/10.3944/aott.2011.2403

3. Barton $\mathrm{C}$ J, Bonanno D, Levinger P, et al. Foot and ankle characteristics in patellofemoral pain syndrome: a case control and reliability study. The Journal of orthopaedic and sports physical therapy (2010) 40(5):286-296. PMID:20436240. https://doi.org/10.2519/jospt.2010.3227

4. Barton C J, Lack S, Hemmings S, et al. The 'Best Practice Guide to Conservative Management of Patellofemoral Pain': incorporating level 1 evidence with expert clinical reasoning. Br J Sports Med (2015) 49(14):923-934. PMID:25716151. https://doi.org/10.1136/bjsports-2014-093637

5. Boling M C, Padua D A, Marshall S W, et al. A prospective investigation of biomechanical risk factors for patellofemoral pain syndrome: the Joint Undertaking to Monitor and Prevent ACL Injury (JUMP-ACL) cohort. The American journal of sports medicine (2009) 37(11):2108-2116. PMID: 19797162 https://doi.org/10.1177/0363546509337934

6. Bramah C, Preece S J, Gill N, et al. A $10 \%$ Increase in Step Rate Improves Running Kinematics and Clinical Outcomes in Runners With Patellofemoral Pain at 4 Weeks and 3 Months. The American journal of sports medicine (2019) 47(14):3406-3413. https://doi.org/10.1177/0363546519879693

7. Chang W-D, Chen F-C, Lee C-L, et al. Effects of Kinesio Taping versus McConnell Taping for Patellofemoral Pain Syndrome: A Systematic Review and Meta-Analysis. Evidence-based complementary and alternative medicine: eCAM (2015) 2015:471208. PMID:26185517. https://doi.org/10.1155/2015/471208

8. Chevidikunnan M F, Al Saif A, Gaowgzeh R A, et al. Effectiveness of core muscle strengthening for improving pain and dynamic balance among female patients with patellofemoral pain syndrome. Journal of physical therapy science (2016) 28(5):1518-1523. PMID:27313363. https://doi.org/10.1589/jpts.28.1518

9. Collins N J, Barton C J, Van Middelkoop M, et al. 2018 Consensus statement on exercise therapy and physical interventions (orthoses, taping and manual therapy) to treat patellofemoral pain: recommendations from the 5th International Patellofemoral Pain Research Retreat, Gold Coast, Australia, 2017. Br J Sports Med (2018) 52(18):1170-1178. PMID:29925502. https://doi.org/10.1136/bjsports-2018-099397

10. Crossley K M, Stefanik J J, Selfe J, et al. 2016 Patellofemoral pain consensus statement from the 4th International Patellofemoral Pain Research Retreat, Manchester. Part 1: Terminology, definitions, clinical 
examination, natural history, patellofemoral osteoarthritis and patient-reported outcome measures. Br J Sports Med (2016) 50(14): 839-843. PMID:27343241. https://doi.org/10.1136/bjsports-2016-096384

11. Dos Santos A F, Nakagawa T H, Lessi G C, et al. Effects of three gait retraining techniques in runners with patellofemoral pain. Physical therapy in sport : official journal of the Association of Chartered Physiotherapists in Sports Medicine (2019) 36. PMID:30703643. https://doi.org/10.1016/j.ptsp.2019.01.006

12. Elias J, Kirkpatrick M, Saranathan A, et al. Hamstrings loading contributes to lateral patellofemoral malalignment and elevated cartilage pressures: an in vitro study. Clinical biomechanics (Bristol, Avon) (2011) 26(8):841-6. PMID:21543144. https://doi.org/10.1016/j.clinbiomech.2011.03.016

13. Finnoff J T, Hall M M, Kyle K, et al. Hip strength and knee pain in high school runners: a prospective study. $P M \& R$ : the journal of injury, function, and rehabilitation (2011) 3(9):792-801.PMID:21821478. https://doi.org/10.1016/j.pmrj.2011.04.007

14. Gaitonde D Y, Ericksen A, Robbins R C. Patellofemoral Pain Syndrome. Am Fam Physician (2019) 99(2):88-94. PMID:30633480

15. Glaviano N R, Saliba S A. Immediate Effect of Patterned Electrical Neuromuscular Stimulation on Pain and Muscle Activation in Individuals With Patellofemoral Pain. J Athl Training (2016) 51(2):118-128. PMID:26967547. https://doi.org/10.4085/1062-6050-51.4.06

16. Hamstra-Wright K L, Aydemir B, Earl-Boehm J, et al. Lasting Improvement of Patient-Reported Outcomes 6 Months After Patellofemoral Pain Rehabilitation. J Sport Rehab (2017) 26(4):223-233. PMID:27632841. https://doi.org/10.1123/jsr.2015-0176

17. Hossain M, Alexander P, Burls A, et al. Foot orthoses for patellofemoral pain in adults. The Cochrane database of systematic reviews (2011) CD008402. PMID:21249707. https://doi.org/10.1002/14651858.CD008402.pub2

18. Hott A, Brox J I, Pripp A H, et al. Effectiveness of Isolated Hip Exercise, Knee Exercise, or Free Physical Activity for Patellofemoral Pain: A Randomized Controlled Trial. The American journal of sports medicine (2019) 47(6):1312-1322. https://doi.org/10.1177/0363546519830644

19. Hunter D J, Zhang Y Q, Niu J B, et al. Patella malalignment, pain and patellofemoral progression: the Health ABC Study. Osteoarthritis Cartilage (2007) 15(10):1120-1127. PMID: 17502158

20. Karst G M, Willett G M. Onset timing of electromyographic activity in the vastus medialis oblique and vastus lateralis muscles in subjects with and without patellofemoral pain syndrome. Phys Ther (1995) 75(9):813-823. PMID:7659741

21. Lack S, Barton $\mathrm{C}$, Sohan $\mathrm{O}$, et al. Proximal muscle rehabilitation is effective for patellofemoral pain: a systematic review with meta-analysis. $\mathrm{Br} J$ Sports Med (2015) 49(21):1365-1376. PMID:26175019. https://doi.org/10.1136/bjsports-2015-094723

22. Lee J, Jang K, Kim E, et al. Effects of Static and Dynamic Stretching With Strengthening Exercises in Patients With Patellofemoral Pain Who Have Inflexible Hamstrings: A Randomized Controlled Trial. Sports health (2021) 13(1):49-56. https://doi.org/10.1177/1941738120932911

23. Liao T, Keyak J, Powers C. Runners With Patellofemoral Pain Exhibit Greater Peak Patella Cartilage Stress Compared With Pain-Free Runners. J Appl Biomech (2018) 34(4):298-305 https://doi.org/10.1123/jab.2017-0229

24. Luz B C, Dos Santos A F, De Souza M C, et al. Relationship between rearfoot, tibia and femur kinematics in runners with and without patellofemoral pain. Gait Posture (2018) 61: 416-422. PMID:29475152. https://doi.org/10.1016/j.gaitpost.2018.02.008

25. Maclachlan L R, Collins N J, Matthews M L G, et al. The psychological features of patellofemoral pain: a systematic review. Br J Sports Med (2017) 51(9):732-742. PMID:28320733.

https://doi.org/10.1136/bjsports-2016-096705

26. Maclachlan L R, Matthews M, Hodges $\mathrm{P}$ W, et al. The psychological features of patellofemoral pain: a cross-sectional study. Scand J Pain (2018) 18(2):261-271. PMID:29794307. https://doi.org/10.1515/sjpain-2018-0025

27. Macri E M, Stefanik J J, Khan K K, et al. Is Tibiofemoral or Patellofemoral Alignment or Trochlear Morphology Associated With Patellofemoral Osteoarthritis? A Systematic Review. Arthritis Care Res (Hoboken) (2016) 68(10):1453-1470. https://doi.org/10.1002/acr.22842

28. Martimbianco A L C, Torloni M R, Andriolo B N, et al Neuromuscular electrical stimulation (NMES) for patellofemoral pain syndrome. The Cochrane database of systematic reviews (2017) 12: CD011289. PMID:29231243. https://doi.org/10.1002/14651858.CD011289.pub2

29. Matthews M, Rathleff M S, Claus A, et al. Does foot mobility affect the outcome in the management of patellofemoral pain with foot orthoses versus hip exercises? A randomised clinical trial. Br J Sports Med (2020). PMID:32217524. https://doi.org/10.1136/bjsports-2019-100935

30. Mcconnell J. A novel approach to pain relief pre-therapeutic exercise. J Sci Med Sport (2000) 3(3):325-334. PMID:11101271

31. Mølgaard C M, Rathleff M S, Andreasen J, et al. Foot exercises and foot orthoses are more effective than knee focused exercises in individuals with patellofemoral pain. $J$ Sci Med Sport (2018) 21(1):10-15. PMID:28844333

32. Mølgaard C, Rathleff M S, Simonsen O. Patellofemoral pain syndrome and its association with hip, ankle, and foot function in 16- to 18-year-old high school students: a single-blind case-control study. J Am Podiatr Med Assoc (2011) 101(3):215-222. PMID:21622633. https://doi.org/10.1016/j.jsams.2017.05.019

33. Neal B S, Lack S D, Lankhorst N E, et al. Risk factors for patellofemoral pain: a systematic review and meta-analysis. Br J Sports Med (2019) 53(5):270-281. PMID:30242107. https://doi.org/10.1136/bjsports-2017-098890

34. Pal S, Besier T F, Gold G E, et al. Patellofemoral cartilage stresses are most sensitive to variations in vastus medialis muscle forces. Comput Methods Biomech Biomed Engin (2019) 22(2):206-216. PMID:30596523. https://doi.org/10.1080/10255842.2018.1544629

35. Powers C M, Witvrouw E, Davis I S, et al. Evidence-based framework for a pathomechanical model of patellofemoral pain: 2017 patellofemoral pain consensus statement from the 4th International Patellofemoral Pain Research Retreat, Manchester, UK: part 3. Br J Sports Med (2017) 51(24):1713-1723. https://doi.org/10.1136/bjsports-2017-098717

36. Priore L B, Azevedo F M, Pazzinatto M F, et al. Influence of kinesiophobia and pain catastrophism on objective function in women with patellofemoral pain. Phys Ther Sport (2019) 35:116-121.

PMID:30529861. https://doi.org/10.1016/j.ptsp.2018.11.013

37. Ramskov D, Barton C, Nielsen R O, et al. High eccentric hip abduction strength reduces the risk of developing patellofemoral pain among novice runners initiating a self-structured running program: a 1-year observational study. The Journal of orthopaedic and sports physical therapy (2015) 45(3):153-161. PMID:25627149. https://doi.org/10.2519/jospt.2015.5091

38. Roper J L, Harding E M, Doerfler D, et al. The effects of gait retraining in runners with patellofemoral pain: A randomized trial. Clinical biomechanics (Bristol, Avon) (2016) 35:14-22. PMID:27111879. https://doi.org/10.1016/j.clinbiomech.2016.03.010

39. Schmitz R J, Shultz S J, Nguyen A-D. Dynamic valgus alignment and functional strength in males and females during maturation. J Athl Training (2009) 44(1):26-32. PMID:19180215. https://doi.org/10.4085/1062-6050-44.1.26

40. Son S J, Kim H, Seeley M K, et al. Efficacy of Sensory 
Transcutaneous Electrical Nerve Stimulation on Perceived Pain and Gait Patterns in Individuals With Experimental Knee Pain. Arch Phys Med Rehabil (2017) 98(1):25-35. PMID:27343344.

https://doi.org/10.1016/j.apmr.2016.05.022

41. Talbot L A, Solomon Z, Webb L, et al. Electrical Stimulation Therapies for Active Duty Military with Patellofemoral Pain Syndrome: A Randomized Trial. Mil Med (2020) 185(7-8):e963-e971.

PMID:32248227. https://doi.org/10.1093/milmed/usaa037

42. Thijs Y, Pattyn E, Van Tiggelen D, et al. Is hip muscle weakness a predisposing factor for patellofemoral pain in female novice runners? A prospective study. The American journal of sports medicine (2011) 39(9):1877-1882. PMID:21632979.

https://doi.org/10.1177/0363546511407617

43. White L, Dolphin P, Dixon J. Hamstring length in patellofemoral pain syndrome. Physiotherapy (2009) 95(1):24-8. PMID:19627682. https://doi.org/10.1016/j.physio.2008.05.009
44. Whyte E F, Moran K, Shortt C P, et al. The influence of reduced hamstring length on patellofemoral joint stress during squatting in healthy male adults. Gait Posture (2010) 31(1): 47-51

PMID:19818627. https://doi.org/10.1016/j.gaitpost.2009.08.243

45. Wilson N A, Press J M, Koh J L, et al. In vivo noninvasive evaluation of abnormal patellar tracking during squatting in patients with patellofemoral pain. The Journal of bone and joint surgery. American volume (2009) 91(3):558-566. PMID:19255215. https://doi.org/10.2106/JBJS.G.00572

46. Xu X, Yao C, Wu R, et al. Prevalence of patellofemoral pain and knee pain in the general population of Chinese young adults: a community-based questionnaire survey. $B M C$ Musculoskelet Disord (2018) 19(1):165. PMID:29793456. https://doi.org/10.1186/s12891-018-2083-x

47. Zago J, Amatuzzi F, Rondinel T, et al. Osteopathic Manipulative Treatment Versus Exercise Program in Runners With Patellofemoral Pain Syndrome: A Randomized Controlled Trial. J Sport Rehab (2020) 1-10. PMID:33333491. https://doi.org/10.1123/jsr.2020-0108 